\title{
BLIND ESTIMATION OF TRANSMIT-POWER FOR MULTIPLE WIRELESS SOURCES
}

\author{
Ivan Wang-Hei Ho*, Bong Jun $\mathrm{Ko}^{\dagger}$, Murtaza Zafer ${ }^{\dagger}$ \\ ${ }^{*}$ Dept. of Electrical and Electronic Engineering, Imperial College London, UK, wh.ho@imperial.ac.uk \\ ${ }^{\dagger}$ IBM T. J. Watson Research Center, US, \{bongjun_ko,mzafer\}@us.ibm.com
}

\begin{abstract}
Received power measurements at spatially distributed, passive, monitors contain valuable information on the active wireless transmitters, which can be usefully exploited to make various inferences. In this paper, we study blind estimation of the number of active wireless sources, their transmission powers and their locations in the network based on received power measurements at multiple monitoring nodes, without assuming any prior knowledge or statistical characterization of these parameters. Utilizing geometrical analysis and algorithmic approach we present estimation algorithms for these parameters under signal combination from multiple transmitters. We present useful non-trivial insights regarding such inferences, and also present simulation results verifying the analysis and quantifying the performance of the estimation algorithms.
\end{abstract}

Index Terms-Transmit-power estimation, Wireless

\section{INTRODUCTION}

Transmission power emitted by wireless nodes decay spatially in the environment according to the physical laws of power propagation. As such, the measured received power at spatially distributed locations is highly correlated and contains valuable information on the wireless network. By exploiting this correlation, one can make valuable inferences about the wireless network. In this paper, we explore this concept and utilize received power measurements at multiple trusting monitors to make "blind" estimation of the transmission power of active nodes. Specifically, using only the received power measurements at multiple monitors, we deduce the number of active transmitters and the transmission powers used by them, without assuming any a-priori knowledge of the locations of the transmitters or any statistical distribution of their transmit powers.

Estimation of transmit power of wireless sources has applicability in a multitude of scenarios such as passive monitoring of wireless networks, user detection in cognitive radio systems and event detection in sensor networks. Passive transmit-power monitoring of a wireless network is important in detecting abnormal or malicious behavior of nodes that can cause excessive signal interference, thereby, causing signal jamming-attack [1] or channel capturing [2]. In cognitive radio, transmit-power estimation can be used to better estimate the interference levels caused by the active users, and the regions where secondary user transmissions would be permissible. In sensor networks, transmit power estimation can be used to identify multiple transient events and their intensities.

In this paper, we consider the following setup: There are multiple wireless sources, and a-priori the number of transmitters, their transmission powers and locations are not known. There are a set of monitors at known locations, each of which measures the 'combined' signal from the transmitters. Note that since we are utilizing only the received signal strength information, it is implicitly assumed that the received signals from different sources are indistinguishable from each other. For the purposes of simplicity, we assume that the power decays deterministically with distance according to the power law. Under this setup, we present algorithms to estimate the number of wireless sources, the transmission power of each source and the locations of active transmitters. We present simulation results quantifying the performance of our algorithms and also point out the limitations.

The above setup is unique in important aspects. First, we consider only passive received power measurements which can be done very easily using simple techniques at the receiver; thus requiring no knowledge of the underlying protocols used by the wireless system. Second, the estimation is done in a blind way, wherein no prior knowledge is assumed about the transmission powers, the locations of the wireless sources, or even the number of sources.

The problem that we consider is unique also from much of the research work in the literature, hence, there is no direct solution applicable to our scenario. In sensor networks, there is an increasing body of literature that deals with sensor node localization [3], [4], [5], [6]. The main problem here is to locate all the nodes in the network based on inter-node distance measurements and using known location information for a small set of nodes, referred to as anchor nodes. A significant difference with this work is that in our case the number of sources and their transmit powers are unknown a-priori. Another related work is MAC layer monitoring wherein specific characteristics of the MAC layer protocol are utilized to monitor wireless nodes' behaviors, for instance, inferring the number of contending nodes [9], finding and isolating selfish nodes [7], [8]; however, in our setup no protocol specific information is considered. 
In preliminary work in [10], we studied the geometric aspects of cooperative power estimation of a single transmitter with up to three monitors. We provide in [11] a more thorough investigation of the problem under wireless fading, giving a formal estimator design, geometrical insights, asymptotic optimality and numerical results based on a data-set of field measurements. While our prior work considered the scenario when the monitors can "decompose" the received signal into the ones from individual transmitters, in this paper we explore the system model where each monitor can only measure the combined signal strength from multiple transmitters.

The rest of the paper is organized as follows. Section II introduces the system model and the two problems (estimations of the number of transmitters and their transmit-powers) that we aim to solve. Section III presents the methodologies and approaches for solving the two problems. Numerical results for evaluating our estimation strategies are presented in Section IV. Finally, Section V concludes the paper.

\section{System MODEL}

We consider a wireless network consisting of a set of $K$ transmitters (or 'sources'), $\left\{t_{1}, t_{2}, \cdots, t_{K}\right\}$, whose locations in the two-dimensional space are $\left(x_{1}, y_{1}\right), \cdots$, $\left(x_{K}, y_{K}\right)$, respectively. The transmit power of the source $t_{i}$ is respectively denoted by $P_{i}$. We assume $K,\left\{P_{i}\right\}$, and $\left\{\left(x_{i}, y_{i}\right)\right\}$ are all unknown.

Also there are a set of $N$ monitors, $\left\{m_{1}, m_{2}, \cdots, m_{N}\right\}$, whose locations are known and denoted by $\left\{\left(\hat{x}_{j}, \hat{y}_{j}\right)\right\}$ for $j=1, \cdots, N$. Henceforth we use the notation $i$ to index the transmitters and $j$ to index the monitors.

We denote by $\hat{P}_{i, j}$ the received power of $t_{i}$ 's signal at monitor $m_{j}$. We assume all monitors are placed in the same wireless transmission medium of all sources, so that each monitor can receive signals transmitted by all sources, i.e., $\hat{P}_{i, j}$ is positive, though it can be arbitrarily small, for all pairs $(i, j)$.

However, at each monitor $m_{j}$, the received signal from all sources are added up together to generate the (combined) measured power value, $\hat{P}_{j}=\sum_{i=1}^{K} \hat{P}_{i, j}$. In other words, the monitors cannot distinguish the received signals from different sources, but can only measure the combined signal strength.

In this paper, we consider the deterministic signal propagation model, where the received power decays in the power of distance between the source and the receiver, i.e.,

$$
\hat{P}_{i, j}=\frac{P_{i}}{d_{i, j}^{\alpha}}
$$

where $d_{i, j}=\left(\left(x_{i}-\hat{x}_{j}\right)^{2}+\left(y_{i}-\hat{y}_{j}\right)^{2}\right)^{1 / 2}$ is the Euclidean distance between the source $i$ and the receiver $j$, and path-loss exponent $\alpha$ is assumed to be a known constant, typically between 2 and 6 . We use the above deterministic model to achieve the fundamental insights on our multitransmitter power estimation problem, and to establish a foundation for future work that can consider more sophisticated assumptions such as wireless fading, unknown signal decay exponents, etc.

According to the above propagation model, the measurement values at the monitors from $K$ transmitters are

$$
\hat{P}_{j}=\sum_{i=1}^{K} \frac{P_{i}}{d_{i, j}^{\alpha}}, \quad \text { for } j=1, \cdots, N,
$$

where $K, P_{i}, d_{i, j}$ for $i=1, \cdots, K$ are all unknown.

Given $N$ received power values $\hat{P}_{j}$ and monitors' locations $\left\{\left(\hat{x}_{j}, \hat{y}_{j}\right)\right\}$ for $j=1, \cdots, N$, one would be interested in finding out, 1) how many transmitters there are (i.e., what is $K$ ?), and 2) the transmission power of each of the $K$ transmitters (i.e., what are $P_{i}$ 's?), that satisfy the set of $N$ equalities in Eq. (2).

Note that, however, given the fixed number of monitors, if there exists a $k$-source solution for Eq. (2), then there exists some solution of $k^{\prime}>k$ sources. Therefore, our goal in this paper is to find out

- the lower bound $k_{\min }$ of $K$, (i.e., at least how many transmitters there should be), and;

- the transmit powers of $k$ sources, $P_{i}, i=1, \cdots, k$, when $k=k_{\min }$,

which satisfy the $N$ equations in Eq. (2).

We call in this paper the first goal the MIN-NUM-TX problem, and the second one the POWER-ESTIMATE $(k)$ problem.

\section{Estimation Method}

In this section, we study the problem of (at least) how many transmitters there should be given the measured received power at $n$ monitors. We first begin with a special case for which we can find an analytic, closed-form solution to the decision version of MIN-NUM-TX for finding out whether there are more than one transmitters. Then we provide a generic approach to determining the minimum number of transmitters.

\section{A. Number of Source Estimation: Geometric Approach}

The decision problem, MIN-NUM-TX- $k$, is to determine whether there are more than $k$ transmitters, from which the solution for MIN-NUM-TX can be obtained by $k_{\text {min }}=$ arg $\min _{k}\{$ MIN-NUM-TX- $k=$ TRUE $\}$. In this subsection, we handle a special case of MIN-NUM-TX-1, i.e., whether 


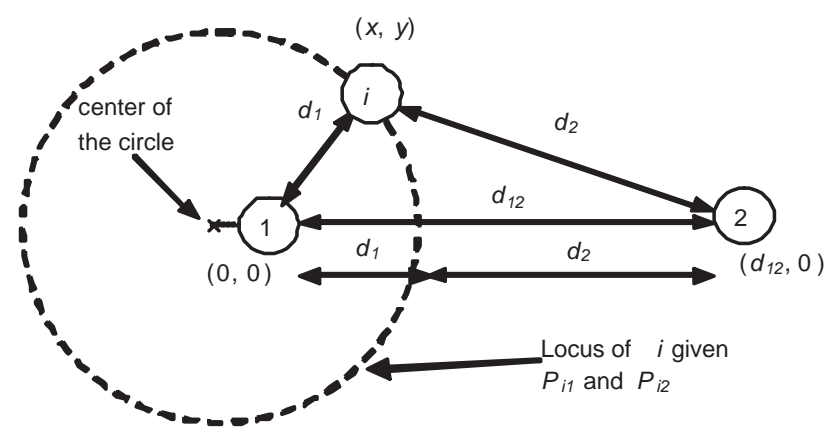

Fig. 1. Schematic diagram showing the circular trajectory of the transmitter given received power measurements at two monitors.

there are more than one transmitters, and provide a solution based on a geometric approach.

The geometric solution for MIN-NUM-TX-1 can be summarized as follows: Suppose we have the received power values at some monitors. We first make an hypothesis that these monitored values are generated by a single transmitter. Then under this hypothesis, we draw the geometry of the possible location(s) of the (assumed) single transmitter based on the received powers. If this geometry indicates that there exists some location of the transmitter satisfying all measurement values, then we conclude that MIN-NUMTX-1 = TRUE, i.e., the minimum number of transmitters is one. Otherwise, we say MIN-NUM-TX-1 = FALSE, i.e., there must be more than one transmitters.

Let us begin with the case that there are only two monitors. According to Proposition 1 in [10], given two distinct received signal power levels observed by a pair of monitors, the possible locations of the transmitter has a circular trajectory (Figure 1), which is drawn from the ratio of those two received power values. Therefore, given any pair of received power levels $\left(\hat{P}_{1}, \hat{P}_{2}\right)$ (from any number of transmitting sources), we can always find the solution for a single transmitter's location, leading to the (quite possibly wrong) conclusion that the hypothesis that there is a single transmitter is correct.

With three monitors, we may or may not be able to identify multiple sources, depending on the locations of the transmitters with respect to the monitors. Consider the case with two transmitters and three monitors. Let the coordinates of monitor 1 be $(0,0)$, the coordinates of monitor 2 be $\left(x_{2}, 0\right)$ and the coordinates of monitor 3 be $\left(x_{3}, y_{3}\right)$. And let us denote the distance between monitors $i$ and $j$ be $d_{i j}$. Therefore, $d_{12}=x_{2}, d_{13}=\left(x_{3}^{2}+y_{3}^{2}\right)^{1 / 2}$.

Let $c_{1}=\left(\frac{\hat{P}_{2}}{\hat{P}_{1}}\right)^{1 / \alpha}$ and $c_{2}=\left(\frac{\hat{P}_{3}}{\hat{P}_{1}}\right)^{1 / \alpha}$ be the ratios between the measured received powers. Under the hypothesis that there is only one signal source unless specified, the algorithm in [10] can be used to identify the position as well as transmit power of such (single) source. Thus, based on these two ratios, two circles can be drawn, and their equations are respectively

$$
\begin{array}{ll}
C 1: \quad & \left(1-c_{1}^{2}\right) x^{2}+\left(1-c_{1}^{2}\right) y^{2} \\
& +2 c_{1}^{2} d_{12} x-c_{1}^{2} d_{12}^{2}=0 \\
C 2: \quad & \left(1-c_{2}^{2}\right) x^{2}+\left(1-c_{2}^{2}\right) y^{2} \\
& +2 c_{2}^{2} x_{3} x+2 c_{2}^{2} y_{3} y-c_{2}^{2} d_{13}^{2}=0
\end{array}
$$

If these two circles intersect, there exists a solution of the possible location of the single transmitter. As shown in [10], with three monitors, these two intersecting circles must intersect at two points, and therefore 'another' solution also exists. In this case, the monitors draw the conclusion that there is only one transmitter located on either points of these two solutions. This conclusion, however, can be wrong in some cases since multiple transmitters can also leave the received power levels of three monitors that result in the intersection of these two circles. On the other hand, if these two circles do not have any intersections (i.e., no possible locations of the single transmitter exist), the three monitors now definitely know that presuming one single signal source is wrong, and can conclude that the received signals must be actually coming from multiple transmitters.

As a result, the problem of determining whether there are solutions for a single transmitter can be transformed to the one of determining if there are intersections between the circles as the following algorithm:

Let $R_{1}$ and $R_{2}$ denote the radius of $C_{1}$ and $C_{2}$, respectively, and let $d$ be the distance between the centers of $C_{1}$ and $C_{2}$.

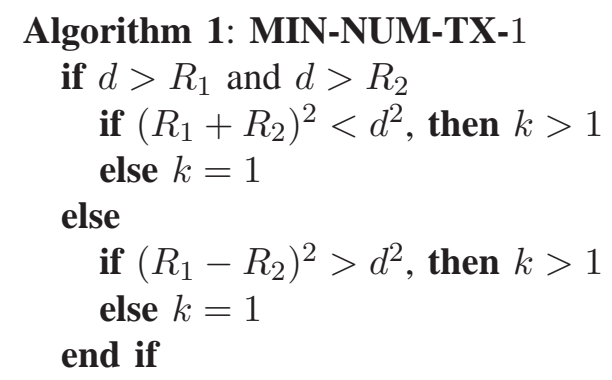

The above algorithm can be used to determine whether there are more than one transmitters with the received power values at three monitors. But the remaining question is: in what case the above algorithm gives you the correct answer? We explore its answer by looking at the geometry of the so-called 'detectable region' as follows.

Let us assume that we are given the location $\left(x_{A}, y_{A}\right)$ of a transmitter (node $A$ ), we are going to explore the region defined by the locations of the other transmitter (node $B$ ) 


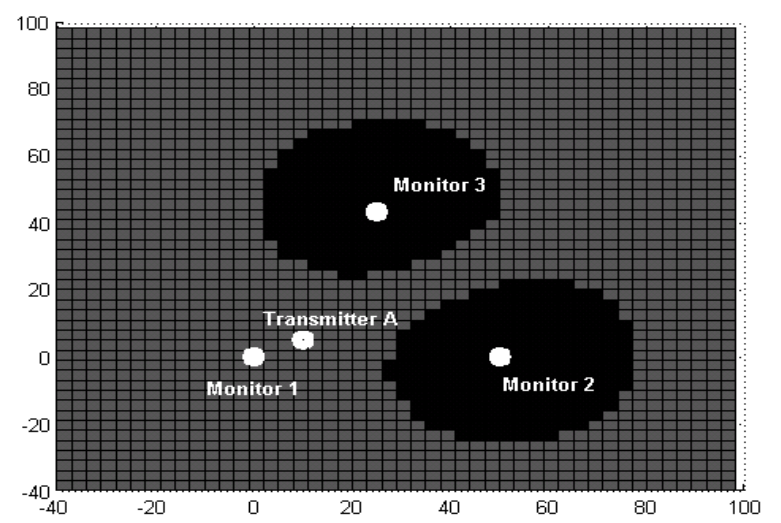

Fig. 2. The detectable region for the position of transmitter $B$ given the positions of the three monitors and transmitter $A$.

that leads to non-intersection circles. We denote this region as the detectable region, where the monitors can identify that the received signal is from more than one sources.

When $d>R_{1}$ and $d>R_{2}, c_{1}<1$ and $c_{2}<1$,

$\left(R_{1}+R_{2}\right)^{2}-d^{2}=\frac{c_{1}^{2} d_{12}^{2}}{1-c_{1}^{2}}+\frac{c_{2}^{2} d_{13}^{2}}{1-c_{2}^{2}}+\frac{c_{1} c_{2}\left(2 d_{12} d_{13}+c_{1} c_{2}\left(d_{12}^{2}+d_{13}^{2}-d_{23}^{2}\right)\right)}{\left(1-c_{1}^{2}\right)\left(1-c_{2}^{2}\right)}$.

Consider a special case that $d_{12}=d_{13}=d_{23}$, then there are no possible solutions for one transmitter if

$$
\frac{\left(c_{1}+c_{2}\right)^{2}-\left(c_{1} c_{2}\right)^{2}}{\left(1-c_{1}^{2}\right)\left(1-c_{2}^{2}\right)}<0 .
$$

The LHS of (5) is regarded as the discriminant for the existence of the solution: If it is less than zero, there are no solutions for single transmitter. (Otherwise, there is solution).

Figure 2 illustrates geometrically an example of the detectable region drawn via numerical simulation. In the figure, the darkest area represents the detectable region of the second transmitter, node B, when the location of the first transmitter (node $\mathrm{A}$ ) is given. It can be seen that, when the transmitter $A$ is closer to one of the monitors (monitor 1 in the figure) than the others, the detectable region corresponds to regions around the other two monitors. It turns out that the closer is the transmitter node A to one of the monitors, the larger becomes the detectable region. As a side note, the detectable region will also shrink as the difference between the two transmit powers $P_{A}$ and $P_{B}$ increases.

With four or more monitors, the existence of singletransmitter solutions is determined by the existence of common intersections of any three of the circles that do not have any triangular dependency. Our conjecture is that the cooperation among four or more number of monitors is capable to detect the existence of multiple sources under the deterministic model (i.e., with full detectable region).
Though we have not been able to verify this conjecture rigorously, our simulation result shows this is the case (i.e., $100 \%$ accuracy with 4 monitors); the reader is referred to Section IV.A for the numerical results.

We note that this geometrical approach can be extended also in the case when the signal decay exponent $\alpha$ is unknown. For instance, when $\alpha$ is only known to be in some range, $\left[\alpha_{\min }, \alpha_{\max }\right]$, the possible locations of the transmitter inferred from two monitors' measurement (in Figure 1) become a torus instead of a circle. Thus the required modification would be that one needs to find if there are intersections of two or more tori to determine whether there can be single transmitter for the given measurement values.

\section{B. Generic Solution to Number of Source Estimation}

Now we provide a solution to MIN-NUM-TX problem for general number of transmitters, in which our goal is to determine whether there are at least $k_{\text {min }}$ transmitters. In principle, if we have an ideal solution to the decision problem MIN-NUM-TX- $k$ that can (correctly) tell whether there are more than $k$ transmitters, $k_{\min }$ can be correctly decided (i.e. $k_{\text {min }}=K$ ) by $k_{\text {min }}=\arg \min _{k>0}\{$ Output(MINNUM-TX- $k)=$ TRUE $\}$.

Note, however, the ability of a monitoring system in providing the solution to MIN-NUM-TX problem is theoretically limited by the available number of monitors $N$. Specifically, in order to correctly determine whether there are at least $K$ transmitters, the monitoring system requires at least $3(K-1)+1$ monitors. This is because if there are only $3(K-1)$ monitors, whose measured received powers constitute a system of $3(K-1)$ equations, one can find a solution that consists of $3(K-1)$ variables for $K-1$ transmitters $\left(P_{i}, x_{i}\right.$, and $y_{i}$, for $\left.i=1, \cdots, k-1\right)$. This means, if there are $K$ transmitters but less than $3(K-1)+1$ monitors, one can always determine (incorrectly) that $k_{\min }=K-1$ due to insufficient information.

Therefore, given $N$ monitors and the corresponding $N$ equations in Eq. (2), our 'best' estimate $k_{\min }^{*}$ is the smallest $k, k=1,2, \cdots$, such that there exists a feasible solution $\left\{\left(P_{i}, x_{i}, y_{i}\right), i=1, \cdots, k\right\}$ to (2). In principle, such $k_{\text {min }}^{*}$ would correctly indicate the actual number of transmitters $K$ given enough number of monitors (i.e., $N>3(K-1)$ ), while it would 'under-estimate' $K\left(k_{\min }^{*}<K\right)$ if there are less monitors than required (i.e., when $N \leq 3(K-1)$ ). Note that, in a special case that it is known a-priori $K \leq$ 2 , the analytic solution in Section III-A correctly outputs $k_{\min }^{*}=1$ or 2 .

However, the non-linearity (especially due to $\alpha \geq 2$ ) makes it intractable to find a closed-form solution to Eq. (2) for general $k>2$. We found that even numerical approaches based on optimization criteria are quite often unable to find 
a feasible solution to all equalities of (2). Hence, we here provide a numerical searching strategy based on a relaxed inequality constraint for the feasibility (as opposed to the strict equality constraint of (2)).

Specifically, given $\hat{P}_{j},\left\{\left(\hat{x}_{j}, \hat{y}_{j}\right)\right\}, j=1, \cdots, n$, we say $\left\{\left(P_{i}, x_{i}, y_{i}\right), i=1, \cdots, k\right\}, P_{i}>0$ for $i=1, \cdots, k$, is $\epsilon$-feasible if

$$
\sum_{j=1}^{n}\left(\hat{P}_{j}-\sum_{i=1}^{k} \frac{P_{i}}{d_{i, j}^{\alpha}}\right)^{2}<\epsilon,
$$

where a constant $\epsilon \geq 0$ is a design parameter. In other words, we allow some margin of quantity $\epsilon$ to the squaresum of $n$ equations of Eq. (2) for the feasibility condition. Then our estimate $k_{\min }^{*}$ is the least $k$ such that there exists an $\epsilon$-feasible solution with $k$-transmitter.

The effect of introducing the $\epsilon$ margin on the performance of the $k_{\text {min }}$ estimator is clear: a large $\epsilon$ allows more data points to satisfy (6), hence making the numerical search to a feasible solution 'easier'. However, a negative impact is that, if $\epsilon$ is too high, some data points of $k^{\prime}$ transmitters for $k^{\prime}<K$ may be found $\epsilon$-feasible, making us 'under-estimate' the minimum number of transmitter (even with sufficient number of monitors). On the other hand, a small $\epsilon$ would make the under-estimating case $k_{\text {min }}^{*}<K$ unlikely, whereas it can make it difficult to find even a legitimate solution $k_{\text {min }}^{*}=K$, leading us to 'over-estimate' $K$ (especially when $N$ is large).

While we recognize the importance of choosing $\epsilon$ for a good estimation quality, we leave a rigorous investigation to it as a future research topic. In our performance evaluation in Section IV, we used a fixed value for $\epsilon$.

\section{Estimating Transmit Powers}

Having presented how to estimate the number of sources, we now proceed to our second problem POWER$\operatorname{ESTIMATE}(k)$ of estimating the transmit powers of $k$ sources. Now that we notice solving the set of $N$ equations of Eq. (2) is analytically intractable for general $k$, our estimate of $P_{i}$ is based on numerical optimization.

Specifically, given $k$, let $\theta$ be the unknown vector of $3 k$ parameters $P_{i}, x_{i}, y_{i}, i=1, \cdots, k$, and $\Omega$ be the set of all admissible values for $\theta$. Then given $k, \hat{P}_{j},\left\{\left(\hat{x}_{j}, \hat{y}_{j}\right)\right\}$, $j=1, \cdots, N$, our estimate $\theta^{*}$ is the optimal one for the following least-square optimization problem:

$$
\begin{gathered}
\theta^{*}=\quad \arg \min _{\theta \in \Omega} \sum_{j=1}^{n}\left(\hat{P}_{j}-\sum_{i=1}^{k} \frac{P_{i}}{d_{i, j}^{\alpha}}\right)^{2}, \\
\text { s.t. } \quad P_{i} \geq 0, \quad i=1, \cdots, k .
\end{gathered}
$$

Note that the above optimization provides the estimate $\theta^{*}$ which consists of the optimal location $\left(x_{i}^{*}, y_{i}^{*}\right)$ as well as $P_{i}^{*}$ for $i=1, \cdots, k$. In other words, given $N$ monitors' locations and their received power values, we also estimate the locations of multiple sources along with their transmit powers. This means our method can be utilized for localizing positions of multiple wireless sources with additional unknown parameters of their transmit powers.

\section{NUMERICAL ANALYSIS}

In this section, we present the performance evaluation result of our estimation methods using numerical analyses on synthetic dataset. The dataset is generated as follows.

For a given number of transmitter, $K$, we pick uniformly at random the locations of $K$ transmitters in a square-area $[-S, S] \times[-S, S]$, with $S=10$, and select the transmit power of each transmitter uniformly at random in $\left[0, P_{\max }\right]$ where $P_{\max }=100$. We generate 100 different sets of such $K$ transmitters.

For each set of $K$ transmitters, we also pick a random locations of $N$ monitors within the same area, for $N=$ $1, \cdots, 10$, for which we generate the received power values based on the deterministic path-loss model in Eq. (2).

The quality of our estimation on the number of sources and the transmit powers depends upon the accuracy of the numerical methods for the optimization. However, the nonlinearity of our objective function and $\epsilon$-feasibility constraint makes numerical search susceptible to falling in local minima, prohibiting it from finding the 'true' optimal or feasible solution.

A remedy to this problem is to iterate the optimization multiple times independently. Therefore, in our evaluation below, we introduce a design parameter $R$, which represents the number of independent iterations that the optimal search is performed, with each search starting from randomly chosen initial parameter $\theta_{0}$. For $\epsilon$-feasibility test for MINNUM-TX, the search stops when a feasible solution is found for the first time, while for the power-estimation, we pick the values that result in the smallest objective function out of all $R$ iterations. Essentially, we tradeoff the computational overhead of repeating the search $R$ times with the accuracy of our estimate: we expect the accuracy of our estimation would increase with larger $R$.

\section{A. Number of Sources Estimation}

Here we present preliminary results for $K=2$ for MIN-NUM-TX estimation with $\epsilon=10^{-4}$. We simulated both the geometric and generic approaches introduced in the previous section. Though we consider $K=2$ for both approaches in the simulations, they represent different decision levels. Note that the geometric approach is for 


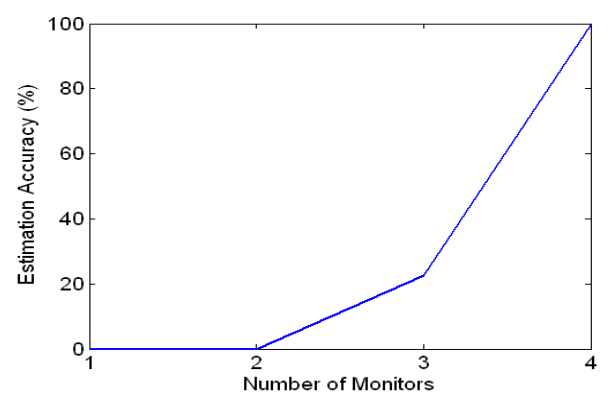

Fig. 3. Estimation accuracy of MIN-NUM-TX-1 geometric approach with $K=2$.

determining whether there is one or more transmitters (i.e., whether $K>1$ ), while the generic approach provides a more general decision of whether there are more than $k$ transmitters (i.e., whether $K>k$, for general $k$ ).

Figure 3 shows the estimation accuracy of the geometric approach (for determining whether $K>1$ ) against the number of cooperating monitors with $K=2$, the result is based on 100 random sets of transmitters and monitors positions. As explained in Section III.A, we can see from the figure that one and two monitors give zero accuracy, three monitors give about $23 \%$ accuracy, and full accuracy can be achieved with four monitors.

Figure 4 shows the performance of our MIN-NUM-TX estimator using $\epsilon$-feasibility test. The three curves in Figure 4(a) depicts the ratio of the transmitter sets (to the total 100 sets), for which the number of monitors in the $x$ axis result in the correct estimation $\left(k_{\min }=K\right)$, underestimation $\left(k_{\min }<K\right)$, and over-estimation $\left(k_{\min }>K\right)$ with $K=2$ after $R=100$ iterations. As we have argued in the previous section, if there are not sufficient number of monitors $(N<4)$, the estimator results mostly in underestimating the number of sources. But as $N$ grows, the cases of correct estimation gets dominating (up to $85 \%$, while there are some under-estimating and over-estimating results. The under-estimation is due to the margin that we give in $\epsilon$-feasibility condition, whereas the over-estimation takes place when the numerical search for the feasible solution fails.

While the under-estimation could be made smaller by having smaller $\epsilon$ margin, this generally comes at the expense of increased over-estimation probability. But The overestimating cases can be suppressed as well by trading it off with the increased computational resources, namely the increased number of search iteration $R$. This is illustrated in Figure 4(b), where it can be seen that the over-estimation probability (when $N$ is large) shrinks down to $5 \%$ as we increase the number search iterations.

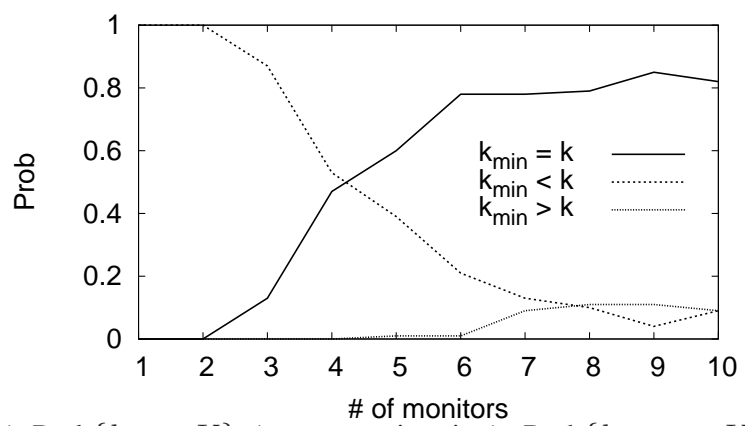

(a) $\operatorname{Prob}\left\{k_{\text {min }}=K\right\}$ (correct estimation), $\operatorname{Prob}\left\{k_{\min }<K\right\}$ (under-estimation), and $\operatorname{Prob}\left\{k_{\min }>K\right\}$ (over-estimation) with $R=100$

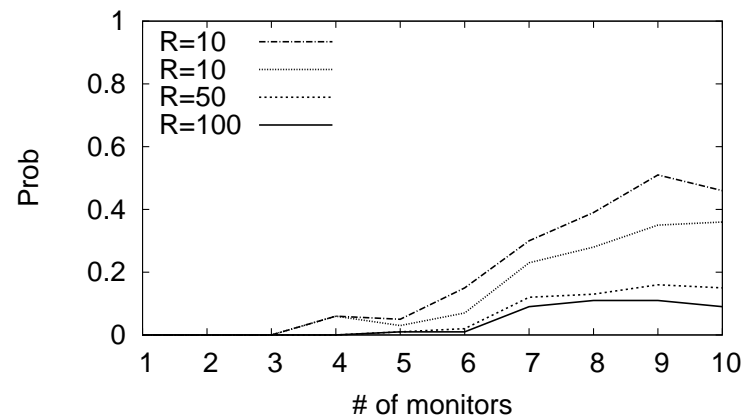

(b) $\operatorname{Prob}\left\{k_{\min }>K\right\}$ (over-estimation) for $R=5,10$, 50,100 . Over-estimation probability decreases with increased search iterations.

Fig. 4. Performance of MIN-NUM-TX estimation $(K=2)$.

\section{B. Transmit-power and Location Estimation}

We now show the performance of the power and location estimation, assuming the correct number of transmitters are given. The result here is obtained for $K=2$. First, we define the power estimation error $\left(P_{e r r}\right)$ and location estimation error $\left(L_{e r r}\right)$ for given number of transmitters $K$ :

$$
\begin{aligned}
& P_{\text {err }}=\frac{1}{K}\left(\sum_{i=1}^{K}\left(\frac{P_{i}^{*}-P_{i}}{P_{i}}\right)^{2}\right)^{1 / 2}, \\
& L_{\text {err }}=\frac{1}{K} \sum_{i=1}^{K} \frac{\left(\left(x_{i}^{*}-x_{i}\right)^{2}+\left(y_{i}^{*}-y_{i}\right)^{2}\right)^{1 / 2}}{2 S} .
\end{aligned}
$$

Note that a normalized term $2 S$ is included in the location error to see the dis-location of the estimated $\left(x_{i}^{*}, y_{i}^{*}\right)$ relative to the the scale of the considered area ( $2 S$ is the side length of the area we generate the transmitters).

Figures 5(a) and 5(b) illustrate how the $P_{\text {err }}$ and $L_{e r r}$, respectively, vary with the number of cooperating monitors. Here we show the results for $N=4$ to 10 as a set of monitors $N<4$ are not likely to estimate the correct number of transmitters as we have seen in Section IV-A. In finding the optimal solution, we added the additional constraints on the location of the transmitters such that $\left(x_{i}, y_{i}\right) \in(-3 S, 3 S) \times(-3 S, 3 S), i=1, \cdots, K$, to avoid solutions converging to prohibitively off-position. Each dot represents the value of $P_{e r r}$ and $L_{e r r}$ of the corresponding 


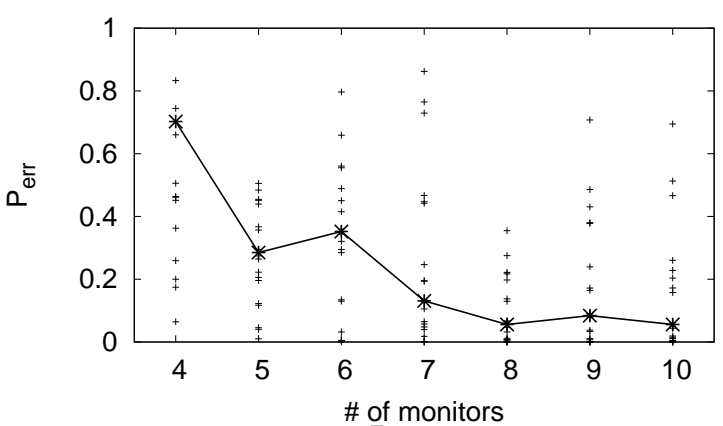

(a) $P_{\text {err }}$

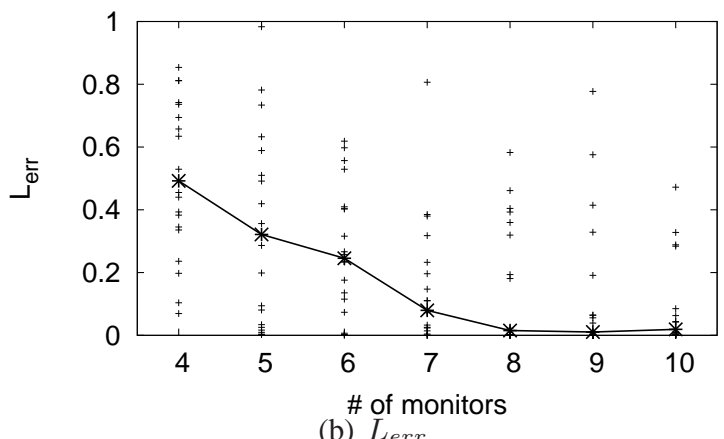

Fig. 5. The accuracy of power and location estimation.

$N$ for each case out of 20 sets of transmitters, and the line connecting the dots across different $N$ shows the median values. We can see that as the number of monitors increases up to ten, both $P_{e r r}$ and $L_{e r r}$ become very small; The estimation quality of transmit-power is within $10 \%$ of the actual value, and the location error is within $2 \%$ of the actual location with respect to the overall length of the area. This is quite an impressive result considering we have not assumed any a-priori knowledge on the power and location (other than the large bound on the location), and once again verifies that the estimation accuracy increases with the number of cooperating monitors.

\section{CONCLUSION}

We studied blind estimation of the number of active transmitters, their transmit-powers and their locations based on received power measurements at spatially distributed monitors, without assuming any prior knowledge of these parameters. Specifically, the setup consisted of a set of passive monitors that measure received power levels from multiple transmitting sources and the goal was to utilize these measurements to estimate the above parameters. We presented geometrical analysis which provided useful insights to the problem. We also proposed estimation algorithms based on a numerical optimization approach and provided illustrative simulation results quantifying the performance of these algorithms. Our work highlights that received power measurements at spatially distributed locations can be utilized to make useful inferences on various network parameters. An important extension of this work in the future will be to investigate the estimation problem under nondeterminstic/uncertan signal propapagation characteristics.

\section{ACKNOWLEDGEMENT}

Research was sponsored by US Army Research laboratory and the UK Ministry of Defence and was accomplished under Agreement Number W911NF-06-3-0001. The views and conclusions contained in this document are those of the authors and should not be interpreted as representing the official policies, either expressed or implied, of the US Army Research Laboratory, the U.S. Government, the UK Ministry of Defense, or the UK Government. The US and UK Governments are authorized to reproduce and distribute reprints for Government purposes notwithstanding any copyright notation hereon.

\section{REFERENCES}

[1] W. Xu, W. Trappe, Y. Zhang, T. Wood, "The feasibility of launching and detecting jamming attacks in wireless networks," ACM MobiHoc, 2005.

[2] I. W.-H. Ho, S. C. Liew, "Impact of Power Control on Performance of IEEE 802.11 Wireless Networks," IEEE Trans. on Mobile Computing, Vol. 6, No. 11, 2007.

[3] N. Patwari, J. Ash, S. Kyperountas, A. O. Hero, R. M. Moses and N. S. Correal, "Locating the Nodes: Cooperative Localization in Wireless Sensor Networks," IEEE Signal Processing Mag., vol. 22, no. 4, July 2005.

[4] C. Savarese, J. M. Rabaey and J. Beutel, "Locationing in distributed ad-hoc wireless sensor networks," in Proc. IEEE ICASSP, May 2001.

[5] R. L. Moses, D. Krishnamurthy, and R. Patterson, "A selflocalization method for wireless sensor networks," EURASIP J. Applied Sig. Proc., no. 4, pp. 348-358, March 2003.

[6] E. G. Larsson, "Cramer-Rao bound analysis of distributed positioning in sensor networks," IEEE Signal Processing Letters,vol. 11, no. 3, March 2004.

[7] P. Kyasanur, N. Vaidya, "Selfish MAC Layer Misbehavior in Wireless Networks," IEEE Trans. on Mobile Computing, Vol. 4, No. 5, 2005.

[8] S. Radosavac, J. Baras, I. Koutsopoulos, "A framework for MAC protocol misbehavior detection in wireless networks," 4th ACM workshop on Wireless security, 2005.

[9] R. Mahajan, M. Rodriget, D. Wetherall, and J. Zahorjan, "Analyzing the MAC-level Behavior of Wireless Networks in the Wild," in Sigcomm 2006.

[10] I. W.-H Ho, B.-J. Ko, M. Zafer, C. Bisdikian, and K. Leung, "Cooperative transmit-power estimation in MANETs," Proc. IEEE WCNC 2008.

[11] M. Zafer, B.-J. Ko and I. W.-H. Ho, "Cooperative Transmit-Power Estimation under Wireless Fading," Proc. ACM Mobihoc 2008, Hong Kong, 2008. 